\title{
Preoperative computed tomography perfusion in pediatric moyamoya disease: a single-institution experience
}

\author{
Vijay M. Ravindra, MD, MSPH, ${ }^{1}$ Stephen F. Kralik, MD, ${ }^{2}$ Julius Griauzde, MD, ${ }^{3}$ Nisha Gadgil, MD, ${ }^{1}$ \\ Melissa A. LoPresti, MD, ${ }^{1}$ and Sandi Lam, MD, MBA ${ }^{4,5}$ \\ 'Division of Pediatric Neurosurgery; ' ${ }^{2}$ epartment of Pediatric Radiology, Baylor College of Medicine, Houston, Texas; ${ }^{3}$ Department \\ of Radiology, University of Michigan, Ann Arbor, Michigan; ${ }^{4}$ Department of Neurosurgery, Northwestern University Feinberg School \\ of Medicine; and ${ }^{5}$ Division of Pediatric Neurosurgery, Ann and Robert H Lurie Children's Hospital, Chicago, Illinois
}

\begin{abstract}
OBJECTIVE Moyamoya disease is a progressive occlusive arteriopathy for which surgical revascularization is indicated. In this retrospective study, the authors investigated the use of preoperative CT perfusion with the aim of establishing pathological data references.

METHODS The authors reviewed the medical records of children with moyamoya disease treated surgically at one institution between 2016 and 2019. Preoperative CT perfusion studies were used to quantify mean transit time (MTT), cerebral blood volume (CBV), cerebral blood flow (CBF), and time to peak (TTP) for the anterior, middle, and posterior cerebral artery vascular territories for each patient. CT perfusion parameter ratios (diseased/healthy hemispheres) and absolute differences were compared between diseased and normal vascular territories (defined by catheter angiography studies). Sensitivity, specificity, and positive (PPV) and negative (NPV) predictive values for CT perfusion parameters for severe angiographic moyamoya were calculated.
\end{abstract}

RESULTS Nine children (89\% female) had preoperative CT perfusion data; 5 of them had evidence of unilateral hemispheric disease and 4 had bilateral disease. The mean age at revascularization was 77 months (range 40-144 months). The etiology of disease was neurofibromatosis type 1 (3 patients), Down syndrome (2), primary moyamoya disease (2), cerebral proliferative angiopathy (1), and sickle cell disease (1). Five patients had undergone unilateral revascularization. Among these patients, pathological vascular territories demonstrated increased MTT in $66 \%$ of samples, increased TTP in $66 \%$, decreased CBF in $47 \%$, and increased CBV in $87 \%$. Severe moyamoya (Suzuki stage $\geq 4$ ) had diseased/healthy ratios $\geq 1$ for MTT in $78 \%$ of cases, for TTP in $89 \%$, for CBF in $67 \%$, and for CBV in $89 \%$. The MTT and TTP region of interest ratio $\geq 1$ demonstrated $89 \%$ sensitivity, $67 \%$ specificity, $80 \%$ PPV, and $80 \%$ NPV for the prediction of severe angiographic moyamoya disease.

CONCLUSIONS Pathological hemispheres in these children with moyamoya disease demonstrated increased MTT, TTP, and CBV and decreased CBF. The authors' results suggest that preoperative CT perfusion may, with high sensitivity, be useful in deciphering perfusion mismatch in brain tissue in children with moyamoya disease. More severe angiographic disease displays a more distinct correlation, allowing surgeons to recognize when to intervene in these patients. https://thejns.org/doi/abs/10.3171/2019.10.PEDS19450

KEYWORDS moyamoya disease; CT perfusion; time to peak; mean transit time; cerebral blood flow; cerebral blood volume; vascular disorders

$\mathrm{M}$ oyamoya disease is a progressive occlusive arteriopathy that is characterized by progressive stenosis and occlusion of the internal carotid artery and its large branches. ${ }^{2}$ In pediatric patients, moyamoya disease typically presents with ischemia due to progressive narrowing and reduction of blood flow. Secondary moy- amoya can occur in the setting of Down syndrome, neurofibromatosis, and sickle cell anemia or after radiation. ${ }^{7}$ Children treated with surgical revascularization, which is standard therapy for pediatric patients with moyamoya, are less likely to have recurrent stroke than those treated medically. ${ }^{4,714}$ 
CT perfusion imaging, a technique used most commonly in the setting of acute ischemic stroke, has also been used to evaluate moyamoya disease, among other cerebrovascular pathologies. ${ }^{5,10,11,16} \mathrm{CT}$ perfusion parameters can be used to evaluate the extent and severity of the angiopathy as well as the need for and urgency of surgical revascularization. Despite the radiation exposure involved with CT perfusion imaging, the widespread availability of this tool coupled with the ability to perform rapid dynamic imaging of the brain (often without the need for sedation) makes it an attractive adjunct for assessing pediatric patients with moyamoya. ${ }^{6}$

In this study, we investigated the use of preoperative $\mathrm{CT}$ perfusion imaging as a diagnostic and predictive tool for the surgical treatment of moyamoya in children. Here, our aim was to report the preliminary results of CT perfusion scanning for diagnoses of perfusion disturbances in children with moyamoya disease.

\section{Methods}

This is a retrospective, nonrandomized observational study of children with moyamoya disease treated surgically at Texas Children's Hospital-Baylor College of Medicine between January 1, 2016, and March 1, 2019. Clinical and radiological data on all patients who had undergone indirect bypass via encephaloduroarteriosynangiosis with dural inversion in this time frame were collected and reviewed. After the MRI and CTA studies, which were performed in all patients, and the DSA studies that were completed once a diagnosis of moyamoya disease had been made, preoperative CT perfusion studies were obtained. The CT perfusion results were analyzed to obtain the mean transit time (MTT), cerebral blood volume (CBV), cerebral blood flow (CBF), and time to peak (TTP). The average values for each parameter were obtained from the anterior and superior anterior cerebral artery (ACA) territories; the anterior, middle, and posterior middle cerebral artery (MCA) territories; and the posterior cerebral artery (PCA) territory for each patient from five mirrored regions of interest (ROIs) in each territory. The absolute difference and ratio of average values for each parameter were compared between normal and diseased hemispheres. Sensitivity, specificity, positive predictive value (PPV), and negative predictive value (NPV) were calculated for CT perfusion ROI ratios of diseased/healthy hemispheres versus the severity of angiographic disease, which was determined in a blinded manner (Suzuki stage).$^{13}$ In each patient, the pathological distribution was used as the benchmark, the MCA in all cases here, yielding three values per patient (anterior MCA, middle MCA, and posterior MCA), with a total of 15 values for each CT perfusion parameter.

\section{Imaging Acquisition}

CT perfusion studies were performed in the axial plane by using a 320-row multidetector CT scanner (Aquilion One, Canon Medical Systems). CT perfusion imaging consisted of a volumetric scan (16-cm craniocaudal coverage) of the entire head in the cine mode during the intravenous administration of iodinated contrast material. A weight-based $(1.5 \mathrm{ml} / \mathrm{kg})$ bolus of nonionic contrast me- dium (Omnipaque, iodine $300 \mathrm{mg} / \mathrm{ml}$, GE Healthcare) was administered into an antecubital vein by using a power injector with an injection rate of 3-4.5 ml/sec, immediately followed by a weight-based saline bolus $(0.75 \mathrm{ml} / \mathrm{kg})$. The CT perfusion acquisition parameters were $80 \mathrm{kVp}, 100$ $130 \mathrm{mAs}, 0.5-\mathrm{mm}$ slice thickness, $0.75 \mathrm{sec} /$ rotation, $22-\mathrm{cm}$ field of view, and image matrix $512 \times 512$.

The CT perfusion parameters of MTT, TTP, CBF, and CBV were generated using Vitrea perfusion software (Vital Images Inc.), and automated mirrored circular ROI placement in both cerebral hemispheres was performed. The cerebral blood perfusion map was calculated with the singular value decomposition. An arterial input function was selected by placing a small circular ROI within the earliest appearing and most densely enhancing artery (usually one of the MCAs ipsilateral to the less affected hemisphere, angiographic stage 1 or 2 , and bolus arrival time difference $<1.7$ seconds). A venous function was selected by placing a circular ROI within a superior sagittal sinus. For each voxel, the CBV map was calculated from the areas under the time-concentration curves. The CBF map for each voxel was finally calculated according to the following equation, which combines the CBV and MTT values: $\mathrm{CBF}=\mathrm{CBV} / \mathrm{MTT}{ }^{1}{ }^{1}$ Calibration for the $\mathrm{CT}$ scanners was performed by a CT technologist and radiologist, and formal interpretation and postprocessing were performed by a neuroradiologist. Figure 1 demonstrates representative images of the technique used.

No anesthesia or sedating medications were required for CT perfusion imaging; the acquisition time was between 1 and 2 minutes (mean 66 seconds). In this series of patients, no acetazolamide challenge was administered given the lack of established protocol and efficacy in the pediatric population.

\section{Statistical Analysis}

Data were summarized using means and standard deviations. Differences and ratios were calculated between diseased and healthy hemispheres. Subsequent cut points were established based on CT perfusion data and clinical relevance, and the use of CT perfusion versus angiography (gold standard) - specifically, the Suzuki stage - was analyzed by calculating sensitivity, specificity, PPV, and NPV. Severe angiographic disease was defined as Suzuki stage 4 or above.

\section{Results}

During the study period, 25 patients underwent cerebral revascularization for moyamoya disease; 9 of them $(89 \%$ female) had preoperative CT perfusion data for analysis. Five of these patients underwent unilateral revascularization and 4 patients underwent bilateral revascularization. The mean age at revascularization was 77 months (range 40-144 months). The etiology of disease was neurofibromatosis type 1 ( $\mathrm{NF} 1 ; \mathrm{n}=3$ patients), Down syndrome ( $\mathrm{n}$ $=2)$, primary moyamoya disease $(n=2)$, cerebral proliferative angiopathy $(n=1)$, and sickle cell disease $(n=1)$. Three children had Suzuki stage 2 disease; 3 children, Suzuki stage 3 ; and 3 children, Suzuki stage 4 . Seven patients had an improvement of 1 point in their modified Rankin 

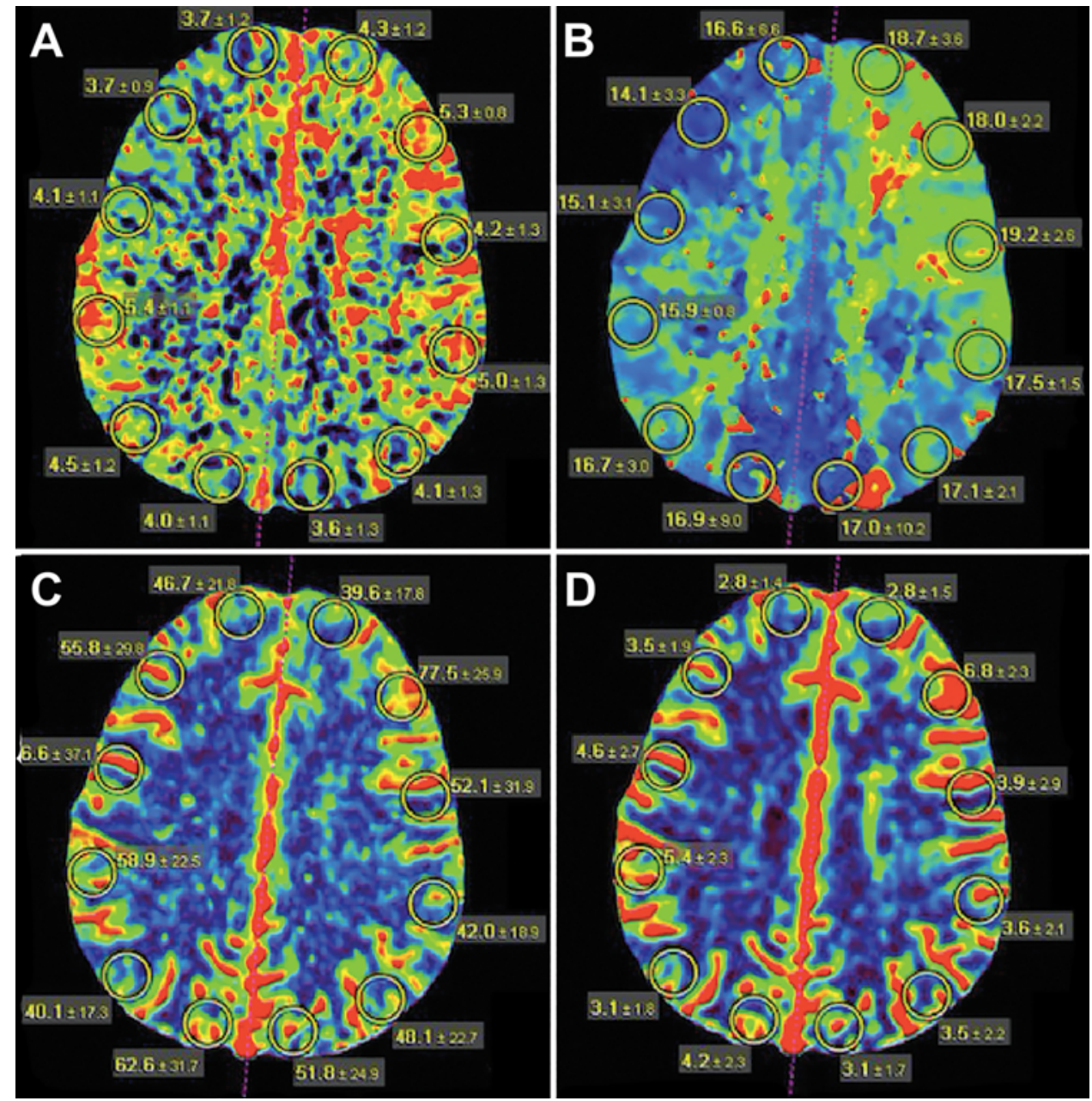

FIG. 1. Representative images obtained in a 12-month-old patient with unilateral moyamoya disease secondary to NF1 (angiographic Suzuki stage 4). CT perfusion maps of MTT, CBV, CBF, and TTP have mirrored ROIs in the right and left cerebral hemispheres. MTT (A) and TTP (B) demonstrated increased transit time for the left cerebral hemisphere. The MCA territory has decreased CBF (C) and increased CBV (D). Figure is available in color online only.

Scale (mRS) score on the last follow-up, 2 patients had no change in their $\mathrm{mRS}$ score, and none of the patients were worse. The mean radiation exposure measured as the CT dose index was $102.5 \mathrm{mGy}$, and the dose length product was $1639.6 \mathrm{mGy}-\mathrm{cm}$.

The 5 patients who had undergone unilateral revascularization served as the study population in which normal and pathological hemispheres were compared. Unilateral disease was defined as no evidence of contralateral disease (ICA, MCA, ACA) on the index image, either CTA or DSA, prior to treatment and before obtaining the CT perfusion study. Four of the 5 patients presented with signs of ischemia, weakness, that resolved. MRI demonstrated small cortical areas of infarction on diffusion imaging corresponding to an MCA distribution. One patient was asymptomatic (Suzuki stage 4). The patient with Suzuki stage 2 disease had a left MCA infarct, with symptoms that resolved.

There was increased MTT in the diseased hemisphere ( $66 \%$ of samples) with a ratio $\geq 1$. For children with severe moyamoya, characterized as Suzuki stage $\geq 4,78 \%$ of val- ues carried a ratio $\geq 1$. The sensitivity for an MTT ROI ratio $\geq 1$ in severe angiographic moyamoya disease was $89 \%$, specificity was $67 \%$, PPV was $80 \%$, and NPV was $80 \%$ (Tables 1 and 2). There was increased TTP in $66 \%$ of samples. In cases of severe moyamoya, $89 \%$ of values had a ratio $\geq 1$. The sensitivity for a TTP ROI ratio $\geq 1$ was $89 \%$, specificity was $67 \%$, PPV was $80 \%$, and NPV was $80 \%$ (Tables 3 and 4 ).

There was decreased CBF in $47 \%$ of samples; however, in severe moyamoya, $67 \%$ of values had a ratio $<1$. The sensitivity for CBF ROI ratio < 1 was $67 \%$, specificity was $83 \%$, PPV was $86 \%$, and NPV was $63 \%$ (Tables 5 and 6). There was increased CBV in $87 \%$ of samples. In cases of severe moyamoya, $89 \%$ of values had a ratio $\geq 1$. Sensitivity for a CBV ROI ratio $\geq 1$ in severe angiographic moyamoya disease was $78 \%$, specificity was $17 \%$, PPV was $58 \%$, and NPV was 50\% (Tables 7 and 8).

\section{Discussion}

In this paper, we described the use of preoperative CT 
TABLE 1. MTT comparisons in patients with unilateral moyamoya disease

\begin{tabular}{|c|c|c|c|c|c|}
\hline $\begin{array}{l}\text { Case } \\
\text { No. }\end{array}$ & $\begin{array}{c}\text { Diseased Hemisphere- } \\
\text { Suzuki Stage }\end{array}$ & $\begin{array}{l}\text { Rt Mean } \\
\text { ROI }\end{array}$ & $\begin{array}{l}\text { Lt Mean } \\
\text { ROI }\end{array}$ & Diff* $^{*}$ & $\begin{array}{c}\text { ROI } \\
\text { Ratio† }\end{array}$ \\
\hline \multirow[t]{7}{*}{1} & Lt MCA-stage 2 & & & & \\
\hline & Ant ACA & 2.5 & 2.3 & -0.2 & 0.92 \\
\hline & Sup ACA & 2.4 & 2.2 & -0.2 & 0.91 \\
\hline & Ant MCA & 2.5 & 2.2 & -0.3 & 0.88 \\
\hline & Mid MCA & 2.4 & 2.3 & -0.1 & 0.96 \\
\hline & Post MCA & 2.5 & 2.3 & -0.2 & 0.92 \\
\hline & PCA & 2.4 & 2.5 & 0.1 & 1.04 \\
\hline \multirow[t]{7}{*}{2} & Rt MCA-stage 3 & & & & \\
\hline & Ant ACA & 3.7 & 3.5 & 0.2 & 1.05 \\
\hline & Sup ACA & 3.4 & 3.7 & -0.3 & 0.92 \\
\hline & Ant MCA & 3.3 & 3.8 & -0.5 & 0.87 \\
\hline & Mid MCA & 3.6 & 3.6 & 0 & 1 \\
\hline & Pst MCA & 3.7 & 3.6 & 0.1 & 1.02 \\
\hline & PCA & 2.6 & 2.8 & -0.2 & 0.93 \\
\hline \multirow[t]{7}{*}{7} & Lt MCA-stage 4 & & & & \\
\hline & Ant ACA & 3.8 & 4.6 & 0.8 & 1.21 \\
\hline & Sup ACA & 4.4 & 4.9 & 0.5 & 1.11 \\
\hline & Ant MCA & 4.1 & 4.1 & 0 & 1 \\
\hline & Mid MCA & 4.0 & 4.8 & 0.8 & 1.2 \\
\hline & Pst MCA & 4.7 & 4.9 & 0.2 & 1.04 \\
\hline & PCA & 3.9 & 4.0 & 0.1 & 1.03 \\
\hline \multirow[t]{7}{*}{8} & Rt MCA-stage 4 & & & & \\
\hline & Ant ACA & 2.5 & 2.3 & 0.2 & 1.03 \\
\hline & Sup ACA & 2.4 & 2.6 & -0.2 & 0.92 \\
\hline & Ant MCA & 3.2 & 2.4 & 0.8 & 1.3 \\
\hline & Mid MCA & 3.3 & 2.3 & 1 & 1.43 \\
\hline & Pst MCA & 3.3 & 2.3 & 1 & 1.43 \\
\hline & PCA & 2.6 & 2.5 & 0.1 & 1.04 \\
\hline \multirow[t]{7}{*}{9} & Lt MCA-stage 4 & & & & \\
\hline & Ant ACA & 2.7 & 2.9 & 0.2 & 1.07 \\
\hline & Sup ACA & 2.7 & 2.9 & 0.2 & 1.07 \\
\hline & Ant MCA & 7.2 & 3.3 & -3.9 & 0.45 \\
\hline & Mid MCA & 2.9 & 3.6 & 0.7 & 1.24 \\
\hline & Pst MCA & 3.0 & 3.4 & 0.4 & 1.13 \\
\hline & PCA & 3.0 & 2.8 & -0.2 & 0.93 \\
\hline
\end{tabular}

Ant $=$ anterior $;$ diff $=$ difference $;$ mid $=$ middle; pst = posterior; sup = superior .

* Difference calculated by subtracting normal from diseased values.

† The ratio of diseased/normal values.

TABLE 2. Comparison of the MTT of the MCA ROI ratio $\geq 1$ and severe moyamoya disease

\begin{tabular}{lccc}
\hline \multicolumn{1}{c}{ Variable } & Suzuki Stage $\geq 4$ & Suzuki Stage $<4$ & Total \\
\hline MCA ROI ratio $\geq 1$ & 8 & 2 & 10 \\
\hline MCA ROI ratio $<1$ & 1 & 4 & 5 \\
\hline Total & 9 & 6 & 15 \\
\hline
\end{tabular}

For an MTT ROI ratio $\geq 1$ in severe moyamoya disease, sensitivity was $89 \%$, specificity was $67 \%$, PPV was $80 \%$, and NPV was $80 \%$.
TABLE 3. TTP comparison in patients with unilateral moyamoya disease

\begin{tabular}{|c|c|c|c|c|c|}
\hline $\begin{array}{l}\text { Case } \\
\text { No. }\end{array}$ & $\begin{array}{c}\text { Diseased Hemisphere- } \\
\text { Suzuki Stage }\end{array}$ & $\begin{array}{l}\text { Rt Mean } \\
\text { ROI }\end{array}$ & $\begin{array}{l}\text { Lt Mean } \\
\text { ROI }\end{array}$ & Diff* & $\begin{array}{c}\text { ROI } \\
\text { Ratio† }\end{array}$ \\
\hline \multirow[t]{7}{*}{1} & Lt MCA-stage 2 & & & & \\
\hline & Ant ACA & 13.2 & 13.1 & -0.1 & 0.99 \\
\hline & Sup ACA & 13.5 & 12.6 & -0.9 & 0.93 \\
\hline & Ant MCA & 13.1 & 12.1 & -1.0 & 0.92 \\
\hline & Mid MCA & 12.7 & 12.4 & -0.3 & 0.97 \\
\hline & Pst MCA & 12.6 & 13.6 & 1.0 & 1.07 \\
\hline & PCA & 14.1 & 14.6 & 0.5 & 1.04 \\
\hline \multirow[t]{7}{*}{2} & Rt MCA-stage 3 & & & & \\
\hline & Ant ACA & 10.4 & 9.6 & 0.8 & 1.08 \\
\hline & Sup ACA & 10.9 & 10.5 & 0.4 & 1.03 \\
\hline & Ant MCA & 9.3 & 10.8 & -1.5 & 0.86 \\
\hline & Mid MCA & 8.9 & 10.4 & -1.5 & 0.85 \\
\hline & Pst MCA & 9.7 & 9.5 & 0.2 & 1.02 \\
\hline & PCA & 7.6 & 7.5 & 0.1 & 1.01 \\
\hline \multirow[t]{7}{*}{7} & Lt MCA-stage 4 & & & & \\
\hline & Ant ACA & 20.5 & 30.2 & 9.7 & 1.47 \\
\hline & Sup ACA & 17.7 & 18.1 & 0.4 & 1.02 \\
\hline & Ant MCA & 15.4 & 19.1 & 3.7 & 1.24 \\
\hline & Mid MCA & 16.9 & 17.5 & 0.6 & 1.04 \\
\hline & Pst MCA & 16.6 & 16.4 & -0.2 & 0.99 \\
\hline & PCA & 22.2 & 15 & -7.2 & 0.68 \\
\hline \multirow[t]{7}{*}{8} & Rt MCA-stage 4 & & & & \\
\hline & Ant ACA & 10.3 & 10.3 & 0 & 1 \\
\hline & Sup ACA & 11.1 & 10.5 & 0.6 & 1.06 \\
\hline & Ant MCA & 12.3 & 10.4 & 1.9 & 1.18 \\
\hline & Mid MCA & 12.1 & 10.1 & 2 & 1.2 \\
\hline & Pst MCA & 11.5 & 10.2 & 1.3 & 1.13 \\
\hline & PCA & 10.4 & 10.5 & -0.1 & 0.99 \\
\hline \multirow[t]{7}{*}{9} & Lt MCA-stage 4 & & & & \\
\hline & Ant ACA & 11 & 9.4 & -1.6 & 0.85 \\
\hline & Sup ACA & 7.8 & 8.8 & 1 & 1.12 \\
\hline & Ant MCA & 8.0 & 9.6 & 1.6 & 1.2 \\
\hline & Mid MCA & 7.3 & 11.2 & 3.9 & 1.53 \\
\hline & Pst MCA & 7.8 & 9.4 & 1.6 & 1.20 \\
\hline & PCA & 9.1 & 9.0 & -0.1 & 0.99 \\
\hline
\end{tabular}

* Difference calculated by subtracting normal from diseased values.

$\dagger$ The ratio of diseased/normal values.

TABLE 4. Comparison of the TTP of the MCA ROI ratio $\geq 1$ and severe moyamoya

\begin{tabular}{lccc}
\hline \multicolumn{1}{c}{ Variable } & Suzuki Stage $\geq 4$ & Suzuki Stage $<4$ & Total \\
\hline MCA ROI ratio $\geq 1$ & 8 & 2 & 10 \\
\hline MCA ROI ratio $<1$ & 1 & 4 & 5 \\
\hline Total & 9 & 6 & 15 \\
\hline
\end{tabular}

For a TTP ROI ratio $\geq 1$ in severe moyamoya disease, sensitivity was $89 \%$, specificity was $67 \%$, PPV was $80 \%$, and NPV was $80 \%$. 
Ravindra et al.

TABLE 5. CBF comparisons in patients with unilateral moyamoya disease

\begin{tabular}{|c|c|c|c|c|c|}
\hline $\begin{array}{l}\text { Case } \\
\text { No. }\end{array}$ & $\begin{array}{c}\text { Diseased Hemisphere- } \\
\text { Suzuki Stage }\end{array}$ & $\begin{array}{l}\text { Rt Mean } \\
\text { ROI }\end{array}$ & $\begin{array}{l}\text { Lt Mean } \\
\text { ROI }\end{array}$ & Diff* $^{*}$ & $\begin{array}{c}\text { ROI } \\
\text { Ratiof }\end{array}$ \\
\hline \multirow[t]{7}{*}{1} & Lt MCA-stage 2 & & & & \\
\hline & Ant ACA & 73.6 & 142.3 & 68.7 & 1.93 \\
\hline & Sup ACA & 98.1 & 144.1 & 46 & 1.46 \\
\hline & Ant MCA & 77.3 & 146.3 & 69 & 1.89 \\
\hline & Mid MCA & 79.4 & 145.1 & 65.7 & 1.82 \\
\hline & Pst MCA & 68 & 106.3 & 38.3 & 1.56 \\
\hline & PCA & 84.6 & 75.7 & -8.9 & 0.89 \\
\hline \multirow[t]{7}{*}{2} & Rt MCA-stage 3 & & & & \\
\hline & Ant ACA & 74.9 & 74.9 & 0 & 1 \\
\hline & Sup ACA & 82.2 & 77.8 & 4.4 & 1.06 \\
\hline & Ant MCA & 77.9 & 67.9 & 10 & 1.14 \\
\hline & Mid MCA & 83.8 & 83.2 & 0.6 & 1.007 \\
\hline & Pst MCA & 72.2 & 72.6 & -0.4 & 0.99 \\
\hline & PCA & 83 & 82.8 & 0.2 & 1.002 \\
\hline \multirow[t]{7}{*}{7} & Lt MCA-stage 4 & & & & \\
\hline & Ant ACA & 41.5 & 57.7 & 16.2 & 1.39 \\
\hline & Sup ACA & 53.4 & 50.7 & -2.7 & 0.95 \\
\hline & Ant MCA & 54 & 60.1 & 6.1 & 1.11 \\
\hline & Mid MCA & 55.3 & 50.6 & -4.7 & 0.92 \\
\hline & Pst MCA & 49.1 & 49.9 & 0.8 & 1.01 \\
\hline & PCA & 51.3 & 62.7 & 11.4 & 1.22 \\
\hline \multirow[t]{7}{*}{8} & Rt MCA-stage 4 & & & & \\
\hline & Ant ACA & 72.4 & 69.3 & 3.1 & 1.045 \\
\hline & Sup ACA & 94.1 & 89.1 & 5 & 1.06 \\
\hline & Ant MCA & 60 & 82.3 & -22.3 & 0.73 \\
\hline & Mid MCA & 64.6 & 90.2 & -25.6 & 0.72 \\
\hline & Pst MCA & 75.5 & 84.1 & -8.6 & 0.90 \\
\hline & PCA & 97 & 85.5 & 11.5 & 1.13 \\
\hline \multirow[t]{7}{*}{9} & Lt MCA-stage 4 & & & & \\
\hline & Ant ACA & 125.9 & 128.9 & 3 & 1.02 \\
\hline & Sup ACA & 127.3 & 128.8 & 1.5 & 1.012 \\
\hline & Ant MCA & 119.7 & 114.7 & -5 & 0.96 \\
\hline & Mid MCA & 128 & 108.3 & -19.7 & 0.85 \\
\hline & Pst MCA & 122.9 & 123.9 & 1 & 1.008 \\
\hline & PCA & 139.9 & 144 & 4.1 & 1.03 \\
\hline
\end{tabular}

* Difference calculated by subtracting normal from diseased values.

$\dagger$ The ratio of diseased/normal values.
TABLE 7. CBV comparisons in patients with unilateral moyamoya disease

\begin{tabular}{|c|c|c|c|c|c|}
\hline $\begin{array}{l}\text { Case } \\
\text { No. }\end{array}$ & $\begin{array}{l}\text { Diseased Hemisphere- } \\
\text { Suzuki Stage }\end{array}$ & $\begin{array}{l}\text { Rt Mean } \\
\text { ROI }\end{array}$ & $\begin{array}{l}\text { Lt Mean } \\
\text { ROI }\end{array}$ & Diff $^{*}$ & $\begin{array}{c}\text { ROI } \\
\text { Ratio† }\end{array}$ \\
\hline \multirow[t]{7}{*}{1} & Lt MCA-stage 2 & & & & \\
\hline & Ant ACA & 3.0 & 6.6 & 3.6 & 2.2 \\
\hline & Sup ACA & 3.9 & 8 & 4.1 & 1.05 \\
\hline & Ant MCA & 3.3 & 7.5 & 4.2 & 2.27 \\
\hline & Mid MCA & 3.7 & 7.2 & 3.5 & 1.95 \\
\hline & Pst MCA & 3.2 & 4.2 & 1 & 1.31 \\
\hline & PCA & 3.9 & 3.3 & -0.6 & 0.85 \\
\hline \multirow[t]{7}{*}{2} & Rt MCA-stage 3 & & & & \\
\hline & Ant ACA & 4.7 & 4.4 & 0.3 & 1.07 \\
\hline & Sup ACA & 4.7 & 4.8 & -0.1 & 0.98 \\
\hline & Ant MCA & 4.6 & 4.3 & 0.3 & 1.07 \\
\hline & Mid MCA & 5 & 5.1 & -0.1 & 0.98 \\
\hline & Pst MCA & 4.5 & 4.5 & 0 & 1 \\
\hline & PCA & 3.6 & 3.9 & -0.3 & 0.92 \\
\hline \multirow[t]{7}{*}{7} & Lt MCA-stage 4 & & & & \\
\hline & Ant ACA & 2.7 & 4.3 & 1.6 & 1.59 \\
\hline & Sup ACA & 3.9 & 4.3 & 0.4 & 1.1 \\
\hline & Ant MCA & 3.7 & 3.7 & 0 & 1 \\
\hline & Mid MCA & 3.8 & 4.1 & 0.3 & 1.08 \\
\hline & Pst MCA & 3.9 & 4.1 & 0.2 & 1.05 \\
\hline & PCA & 3.3 & 4.1 & 0.8 & 1.24 \\
\hline \multirow[t]{7}{*}{8} & Rt MCA-stage 4 & & & & \\
\hline & Ant ACA & 2.9 & 2.6 & 0.3 & 1.12 \\
\hline & Sup ACA & 3.7 & 3.5 & 0.2 & 1.06 \\
\hline & Ant MCA & 3.6 & 3 & 0.6 & 1.2 \\
\hline & Mid MCA & 3.8 & 3.6 & 0.2 & 1.06 \\
\hline & Pst MCA & 3.9 & 3.5 & 0.4 & 1.11 \\
\hline & PCA & 4.3 & 2.9 & 1.4 & 1.48 \\
\hline \multirow[t]{7}{*}{9} & Lt MCA-stage 4 & & & & \\
\hline & Ant ACA & 8.6 & 9.9 & 1.3 & 1.15 \\
\hline & Sup ACA & 8.2 & 8.3 & 0.1 & 1.01 \\
\hline & Ant MCA & 8.3 & 10.1 & 1.8 & 1.22 \\
\hline & Mid MCA & 8.9 & 8.2 & -0.7 & 0.92 \\
\hline & Pst MCA & 7.7 & 8.8 & 1.1 & 1.14 \\
\hline & PCA & 10.1 & 10.7 & 0.6 & 1.06 \\
\hline
\end{tabular}

${ }^{*}$ Difference calculated by subtracting normal from diseased values.

$\dagger$ The ratio of diseased/normal values.
TABLE 6. Comparison of the CBF of the MCA ROI ratio $\geq 1$ and severe moyamoya

\begin{tabular}{lccc}
\hline \multicolumn{1}{c}{ Variable } & Suzuki Stage $\geq 4$ & Suzuki Stage $<4$ & Total \\
\hline MCA ROI ratio $\geq 1$ & 6 & 1 & 7 \\
\hline MCA ROI ratio $<1$ & 3 & 5 & 8 \\
\hline Total & 9 & 6 & 15 \\
\hline
\end{tabular}

For a $\mathrm{CBF}$ ROI ratio $<1$ in severe moyamoya disease, sensitivity was $67 \%$, specificity was $83 \%$, PPV was $86 \%$, and NPV was $63 \%$.
TABLE 8. Comparison of the CBV of the MCA ROI ratio $\geq 1$ and severe moyamoya

\begin{tabular}{lccc}
\hline Variable & Suzuki Stage $\geq 4$ & Suzuki Stage $<4$ & Total \\
\hline MCA ROI ratio $\geq 1$ & 7 & 5 & 12 \\
\hline MCA ROI ratio $<1$ & 2 & 1 & 3 \\
\hline Total & 9 & 6 & 15 \\
\hline
\end{tabular}

For a CBV ROI ratio $\geq 1$ in severe moyamoya disease, sensitivity was $78 \%$, specificity was $17 \%$, PPV was $58 \%$, and NPV was $50 \%$. 
perfusion in children with moyamoya disease at a single center. The overall evaluation of brain perfusion is paramount in moyamoya disease to determine the extent and severity of the resultant angiopathy. ${ }^{6}$ Our results suggest that the CT perfusion parameters of MTT, TTP, CBF, and CBV can be altered in children with moyamoya and may have predictive capability with regard to the angiographic severity of the disease.

To ascertain whether surgical revascularization is needed and the urgency of such revascularization, DSA and assessment of $\mathrm{CBF}$ are required. ${ }^{12} \mathrm{CT}$ perfusion has gained popularity because of its widespread access and ability to rapidly quantify lesions in the setting of adult ischemic stroke. ${ }^{16}$ In this series, we demonstrated that children with unilateral moyamoya disease have a relatively prolonged MTT and TTP in the diseased hemisphere, reduced CBF, and increased CBV. Moreover, CBV apparently increases in the diseased hemisphere as a compensatory mechanism, similar to a penumbra pattern in stroke. We believe the findings represent moyamoya physiology, and we propose that CT perfusion may provide baseline findings and a method of evaluating pediatric patients.

When considering revascularization, the ultimate goal is to determine cerebrovascular reserve capacity (CVRC), which has been shown to have value in quantifying disease severity. 3,17 Thus, the CVRC can be used to identify "at-risk" territory and increase efforts for treatment, either medical or surgical. CVRC can be difficult to quantify in pediatric moyamoya disease because neovascularity along the MCA branches may not specifically affect CVRC, as previously seen in cerebrovascular atherosclerotic disease. Specifically, $\mathrm{CO}_{2}$ or acetazolamide challenge studies are difficult to obtain in pediatric patients, especially those of very young age, because of the lack of a standardized dosing recommendation, the potential need for sedation, and the paucity of institutional protocols for this type of imaging in children.

\section{CT Perfusion in Moyamoya}

Various imaging modalities can be performed to assess cerebral perfusion, including PET, SPECT, xenon CT, and MRI. The advantages of CT perfusion include a short acquisition time, high spatial resolution, high level of accessibility to CT imaging, and automated postprocessing software. In addition, using a 320-row multidetector CT scanner allows isotropic volumetric data of the whole brain from which both CTA and CT perfusion data can be obtained, which can be useful in the evaluation of patients with moyamoya. CT perfusion provides complementary information to the vascular information provided by CTA and/or cerebral catheter angiography. CT perfusion also represents a noninvasive measure of physiological blood flow. CT perfusion is less costly than MR perfusion scanning, is more widely and readily available, and can often be performed without general anesthesia. Additionally, CT perfusion makes multiple passes through ROIs in the brain, thus increasing the sampling and accuracy of the image information. The disadvantages of CT perfusion include ionizing radiation and unsuitability of iodinated contrast in some patients. The CT dose index and dose length product were higher than the mean for children undergoing routine CTA in trauma cases; ${ }^{9}$ however, the effective doses in CT perfusion can be as low as $1.2 \mathrm{mSv}$ if dose reduction strategies are used, ${ }^{15}$ whereas diagnostic cerebral angiography has an estimated effective dose of $2.7 \mathrm{mSv}^{8}$

The use of CT perfusion has been previously described in moyamoya disease, ${ }^{5,10,11,16}$ but only once in a pediatric patient. Sakamoto et al. ${ }^{11}$ described the use of CT perfusion imaging in a 12-year-old girl both pre- and postvascularization. They concluded that CT perfusion imaging was equivalent to SPECT with acetazolamide in terms of accuracy and was superior in spatial resolution; thus, its use was helpful in the management and treatment of pediatric moyamoya.

In this investigation, we found that elevated MTT and TTP correlated with impaired baseline delivery of the contrast material and decreased $\mathrm{CBF}$ and increased $\mathrm{CBV}$. The sensitivity for both the MTT and TTP ROI ratios $\geq 1$ was $89 \%$, indicating an elevated ability to rule out severe angiographic disease with $80 \%$ PPV and NPV. We found an increased $\mathrm{CBF}$ in $53 \%$ of samples; however, in severe moyamoya, $67 \%$ of values had a ratio $<1$. The sensitivity for a CBF ROI ratio < 1 was $67 \%$, specificity was $83 \%$, PPV was $86 \%$, and NPV was $63 \%$. In a cohort study of adult patients with moyamoya disease, Schubert et al. ${ }^{12}$ also found a decreased $\mathrm{CBF}$. The $\mathrm{CBV}$ ratio was $\geq 1$ in $87 \%$ of all samples and in $89 \%$ of values in severe disease. The sensitivity for a CBV ROI ratio $\geq 1$ in severe angiographic moyamoya disease was $78 \%$, again with a high level of reliability in ruling out severe moyamoya disease. With the low specificity of $17 \%$, this parameter could be used as a screening test with DSA as confirmation. The utility of ruling out stage 4 disease comes in the benefit of avoiding the ordering of additional testing, that is, conventional angiography. Regions of reduced perfusion demonstrate prolonged MTT and TTP, which is helpful in identifying the extent of the perfusion abnormality, which, in turn, is helpful in risk-benefit ratio discussions prior to revascularization procedures.

\section{Study Limitations}

This single-center experience with CT perfusion in moyamoya disease has inherent limitations. At our institution, we use a mirrored ROI approach to determine the mean CT perfusion values, and these values were obtained in territories classically supplied by the ACA, MCA, and PCA. Additionally, only patients who had undergone unilateral revascularization for unilateral disease were included in our analysis, although the process is often bilateral in children with moyamoya disease. A comparison with healthy, age-matched children without moyamoya disease was not performed because we conducted a retrospective study from available CT perfusion data. Given similar alterations in CT perfusion parameters in adults with moyamoya, we believe that these results in children with moyamoya are representative of the physiological blood flow alterations and can be useful in guiding when to intervene in asymptomatic children with radiographic findings of moyamoya disease to prevent ischemic or hemorrhagic stroke events. Dynamic CT perfusion can also be used as a screening test given its noninvasive nature, and it can si- 
multaneously provide CTA data that can be used to assess the arterial stenosis before revascularization procedures.

This report suffers from a small sample size; of the 25 patients who had a diagnosis of moyamoya disease and underwent surgery, only 9 had preoperative CT perfusion imaging, and these patients had differing pathologies. This was due to historical variation in individual surgeon practices, which has more recently been standardized with clinical pathways subsequent to the study's data collection dates. Despite the small sample size, this report is the largest documented experience of CT perfusion in pediatric patients to date. The heterogeneity of the age and pathology of the included patients is a byproduct of attempting to increase the sample size and the number of data points possible for meaningful analysis.

An additional limitation is the characterization of Suzuki stage 4 and above as severe. This was chosen as a dichotomy based on the original description of moyamoya disease $^{13}$ and from the data available from our cohort (angiographic severity). With a larger sample size, the differences between stages 3 and 4 may not be as apparent, and this possibility should be further investigated; additionally, having only one patient with grade 3 disease makes extrapolating the findings difficult. The use of CT perfusion may serve as a noninvasive, sensitive screening test that could reduce the number of more invasive studies that would be needed. The risks of radiation and contrast exposure would need to be outweighed by the benefits of the clinically relevant information gained: this question must be further investigated, although this study is an initial step.

Although we explored the use of CT perfusion in moyamoya patients, we do not suggest replacing DSA with CT perfusion plus CTA. Instead, our study provides examples for the use of CT perfusion in potentially asymptomatic patients as a method of quantifying or objectively reporting perfusion deficit. DSA is superior in defining vascular anatomy, although the physiological changes during perfusion are not as demonstrable; thus, CT perfusion in this setting could serve as a screening test. If CT perfusion imaging is suggestive of perfusion mismatch, then a more invasive study, such as DSA, could be performed. To truly define characteristics of an "at-risk" asymptomatic patient, we would need CT perfusion studies in more children with moyamoya disease who go on to have ischemic disease. In the future, a case-control study design including all children with CT perfusion could accomplish this. The current report serves as an initial framework for the use of CT perfusion in moyamoya and the interpretation of pathological values by presenting trends in the experience of a single center. Further perfusion imaging techniques with acetazolamide challenge, transcranial Doppler, or MR perfusion will be important, and direct comparison with CT perfusion will allow assessment of the best choice for perfusion imaging in children.

\section{Conclusions}

We have demonstrated the technique of CT perfusion for assessment of perfusion mismatch in pediatric patients with moyamoya. The CT perfusion parameters correlated with the angiographic severity of the disease in the children who were candidates for cerebral revascularization. These data may be useful in identifying the cerebrovascular reserve available, which can help define the timing of intervention. Further correlation with long-term angiographic outcomes and perioperative complications is necessary.

\section{References}

1. Axel L: Tissue mean transit time from dynamic computed tomography by a simple deconvolution technique. Invest Radiol 18:94-99, 1983

2. Gadgil N, Lam S, Pyarali M, Paldino M, Pan IW, Dauser RC: Indirect revascularization with the dural inversion technique for pediatric moyamoya disease: 20 -year experience. J Neurosurg Pediatr 22:541-549, 2018

3. Gupta A, Chazen JL, Hartman M, Delgado D, Anumula N, Shao H, et al: Cerebrovascular reserve and stroke risk in patients with carotid stenosis or occlusion: a systematic review and meta-analysis. Stroke 43:2884-2891, 2012

4. Guzman R, Lee M, Achrol A, Bell-Stephens T, Kelly M, Do $\mathrm{HM}$, et al: Clinical outcome after 450 revascularization procedures for moyamoya disease. Clinical article. J Neurosurg 111:927-935, 2009

5. Kang KH, Kim HS, Kim SY: Quantitative cerebrovascular reserve measured by acetazolamide-challenged dynamic CT perfusion in ischemic adult moyamoya disease: initial experience with angiographic correlation. AJNR Am J Neuroradiol 29:1487-1493, 2008

6. Lee M, Zaharchuk G, Guzman R, Achrol A, Bell-Stephens T, Steinberg GK: Quantitative hemodynamic studies in moyamoya disease: a review. Neurosurg Focus 26(4):E5, 2009

7. Lee S, Rivkin MJ, Kirton A, deVeber G, Elbers J: Moyamoya disease in children: results from the International Pediatric Stroke Study. J Child Neurol 32:924-929, 2017

8. Manninen AL, Isokangas JM, Karttunen A, Siniluoto T, Nieminen MT: A comparison of radiation exposure between diagnostic CTA and DSA examinations of cerebral and cervicocerebral vessels. AJNR Am J Neuroradiol 33:2038 2042,2012

9. Ravindra VM, Riva-Cambrin J, Sivakumar W, Metzger RR, Bollo RJ: Risk factors for traumatic blunt cerebrovascular injury diagnosed by computed tomography angiography in the pediatric population: a retrospective cohort study. J Neurosurg Pediatr 15:599-606, 2015

10. Rim NJ, Kim HS, Shin YS, Kim SY: Which CT perfusion parameter best reflects cerebrovascular reserve?: correlation of acetazolamide-challenged CT perfusion with single-photon emission CT in moyamoya patients. AJNR Am J Neuroradiol 29:1658-1663, 2008

11. Sakamoto S, Ohba S, Shibukawa M, Kiura Y, Arita K, Kurisu $\mathrm{K}$ : CT perfusion imaging for childhood moyamoya disease before and after surgical revascularization. Acta Neurochir (Wien) 148:77-81, 2006

12. Schubert GA, Czabanka M, Seiz M, Horn P, Vajkoczy P, Thomé C: Perfusion characteristics of moyamoya disease: an anatomically and clinically oriented analysis and comparison. Stroke 45:101-106, 2014

13. Suzuki J, Takaku A: Cerebrovascular "moyamoya" disease. Disease showing abnormal net-like vessels in base of brain. Arch Neurol 20:288-299, 1969

14. Veeravagu A, Guzman R, Patil CG, Hou LC, Lee M, Steinberg GK: Moyamoya disease in pediatric patients: outcomes of neurosurgical interventions. Neurosurg Focus 24(2):E16, 2008

15. Wintermark M, Maeder P, Verdun FR, Thiran JP, Valley JF, Schnyder P, et al: Using $80 \mathrm{kVp}$ versus $120 \mathrm{kVp}$ in perfusion 
CT measurement of regional cerebral blood flow. AJNR Am J Neuroradiol 21:1881-1884, 2000

16. Xie A, Luo L, Ding Y, Li G: Ischemic and hemorrhagic moyamoya disease in adults: CT findings. Int J Clin Exp Med 8:21351-21357, 2015

17. Yonas H, Smith HA, Durham SR, Pentheny SL, Johnson DW: Increased stroke risk predicted by compromised cerebral blood flow reactivity. J Neurosurg 79:483-489, 1993

\section{Disclaimer}

The views expressed herein are those of the author(s) and do not necessarily reflect the official policy or position of the Department of the Navy, Department of Defense, or the U.S. Government.

\section{Disclosures}

The authors report no conflict of interest concerning the materials or methods used in this study or the findings specified in this paper.

\section{Author Contributions}

Conception and design: Ravindra. Acquisition of data: Ravindra, Kralik. Analysis and interpretation of data: Kralik. Drafting the article: Ravindra. Critically revising the article: all authors. Reviewed submitted version of manuscript: all authors. Approved the final version of the manuscript on behalf of all authors: Lam.

\section{Supplemental Information}

\section{Current Affiliations}

Dr. Ravindra: Department of Neurosurgery, Naval Medical Center San Diego, San Diego, California.

\section{Correspondence}

Sandi Lam: Northwestern University Feinberg School of Medicine, Chicago, IL. sandilam@luriechildrens.org. 